J Nucl Cardiol. 2015 December ; 22(6): 1214-1221. doi:10.1007/s12350-014-0050-y.

\title{
The prognostic value of regadenoson myocardial perfusion imaging
}

Fadi G. Hage, MD, FASH, FACC ${ }^{a, b}$, Gopal Ghimire, MD ${ }^{a}$, Davis Lester, BS ${ }^{c}$, Joshua Mckay, MD $^{\mathrm{a}}$, Steven Bleich, MD ${ }^{\mathrm{a}}$, Stephanie El-Hajj, MD ${ }^{\mathrm{d}}$, and Ami E. Iskandrian, MD, MACC ${ }^{\mathrm{a}}$

aDepartment of Medicine, University of Alabama at Birmingham, Birmingham, AL

${ }^{b}$ Birmingham Veterans Affairs Medical Center, Birmingham, AL

'School of Medicine, University of Alabama at Birmingham, Birmingham, AL

dDepartment of Medicine, Louisiana State University, Baton Rouge, LA

\section{Abstract}

Background-Regadenoson (REGA), a selective adenosine $\mathrm{A}_{2 \mathrm{~A}}$ receptor agonist, is the most widely used stress agent for SPECT myocardial perfusion imaging (MPI) in the United States. The diagnostic accuracy of REGA MPI is comparable to Adenosine MPI, but its prognostic value is not well defined.

Methods-We categorized 1,400 patients (700 consecutive normal and 700 consecutive abnormal REGA-MPIs) into 4 groups based on the perfusion defect size using automated quantitative analysis: Group 1: normal perfusion; Group 2: <10\% of left ventricle; Group 3: 10\%20\%; Group 4: $>20 \%$. The primary outcome was a composite of cardiac death, myocardial infarction (MI), and late coronary revascularization (CR >90 days after MPI).

Results-Of the 1,400 patients ( $42 \%$ male, $37 \%$ diabetes, $21 \%$ heart failure, $26 \%$ end-stage renal disease), the primary outcome occurred in $23 \%$ (17\% cardiac death, $4 \%$ MI, $6 \%$ late CR) during $46 \pm 18$ months of follow-up and $8 \%$ had early CR (within 90 days of MPI). Early CR occurred in $0.4 \%, 9 \%, 17 \%$, and $17 \%$ and the primary outcome in $10 \%, 27 \%, 31 \%$, and $43 \%$ in Groups $1-4$, respectively ( $P<.001$ for both). In an adjusted Cox proportional model, the hazard ratio for the primary outcome was 2.68 (1.77-4.06), 3.32 (2.28-4.83), and 4.05 (2.78-5.91) for Groups 2-4 compared to Group 1.

Conclusion-REGA MPI provides powerful prognostic information that has important implications in patient management and can guide clinical practice.

\section{Keywords}

Myocardial perfusion imaging; regadenoson; prognosis; quantitative analysis

Reprint requests: Fadi G. Hage MD, FASH, FACC, Department of Medicine, University of Alabama at Birmingham, Lyons Harrison Research Building 314, 1900 University BLVD, Birmingham, AL 35294; fadihage @uab.edu. 


\section{BACKGROUND}

Regadenoson (REGA), a selective adenosine $\mathrm{A}_{2 \mathrm{~A}}$ receptor agonist, received approval from the Food and Drug Administration in the United States in 2008 based on the results of randomized phase 3 multi-center trials that established its non-inferiority to adenosine for the diagnosis of reversible perfusion abnormalities. ${ }^{1-3}$ Within a short time span, REGA became the most widely used pharmacologic stress agent for SPECT myocardial perfusion imaging (MPI) in the United State accounting for $84 \%$ of the market in $2013.4,5$ The overwhelming adoption of REGA by the nuclear cardiology community is likely dependent on its well-tolerability by patients, its safety profile in many subgroups of patients (including those with reactive airway disease, liver disease, renal disease, and cardiac transplantation), and its ease of administration as a fixed-dose bolus which has greatly simplified stress protocols. ${ }^{1}$

A large body of evidence has accumulated supporting the use of MPI for prognostication, ${ }^{6}$ but despite its widespread use, there is paucity of data on prognostic value of perfusion abnormalities associated with REGA MPI. It is clearly important to establish the prognostic utility of REGA MPI beyond its diagnostic capabilities since it is widely used for risk stratification. We have previously established that major cardiac events are infrequent in patients with normal REGA MPI and that the rate of events in patients with normal REGA MPI did not exceed that encountered in patients with normal adenosine MPI. ${ }^{7}$ In this study, we evaluated the prognostic value of automated quantitation of perfusion abnormalities by REGA MPI.

\section{METHOD}

\section{Study Cohort}

We studied 1,400 patients who underwent pharmacological MPI using REGA at University of Alabama at Birmingham from July 2008 to January 2010 for clinical purposes. We included 700 consecutive patients with normal perfusion and normal left ventricular (LV) systolic function (defined as LVEF $250 \%$ ) on MPI and 700 consecutive patients with abnormal myocardial perfusion (defined as perfusion defect size—perfusion defect size (PDS) $25 \%$ of LV) The study was approved by the institutional review board for human research at the University of Alabama at Birmingham.

\section{MPI Protocol}

REGA MPI was performed using standard protocols approved by the American Society of Nuclear Cardiology. ${ }^{8}$ The specifics of stress testing, and image acquisition and interpretation have been previously published. ${ }^{7}$ Briefly, a single bolus of $0.4 \mathrm{mg}$ of REGA was administered via a peripheral venous access, followed by saline flush. The tracer, technetium-99m-sestamibi, was administered intravenously 10-20 seconds after the saline flush. Gated SPECT images were acquired 1 hour after tracer injection with a dual-head detector gamma camera with a low-energy, high-resolution collimator with a $64 \times 64$ matrix. The cameras operated in an elliptical $180^{\circ}$ acquisition orbit with 32 projections and 30 seconds per projection. For image acquisition, a 15\% energy window focused on the 140- 
$\mathrm{keV}$ gamma peak was utilized. Gating was performed with 8 to 16 frames/RR cycle. Butterworth filtering and filtered back projection reconstruction was done. The image interpretation was performed in the absence of attenuation or scatter correction.

The presence and extent of perfusion abnormalities were determined by a software program (Corridor4DM) with visual supervision. ${ }^{9}$ The readers were blinded to subsequent events. LVEF was determined from stress gated images using previously described methods. ${ }^{10}$ Perfusion defects were quantitated as \% of LV myocardium and expressed as PDS (total perfusion defect), ischemia (reversible perfusion defect), and scar (fixed perfusion defect). Patients were divided into 4 categories based on PDS: Group 1 with no perfusion defect, Group 2 with PDS <10\% LV; Group 3 with PDS 10\%-20\%, and Group 4 with PDS >20\%. Patients were separately divided based on extent of ischemia into 4 Groups: Group 5 with no ischemia, Group 6 with ischemia involving <10\% LV, Group 7 with 10\%-20\%, and Group 8 with $>20 \%$.

\section{Outcome Determination}

The study cohorts were followed for outcomes including cardiac death, myocardial infarction (MI), and coronary revascularization (CR) which included both coronary artery bypass grafting $(\mathrm{CABG})$ and percutaneous coronary interventions $(\mathrm{PCI})$. The outcome events were ascertained by review of patient health records beginning from date of the index MPI to November 2013. Scripted telephonic interviews were used for subjects whose follow-up was less than 2 years. Social Security Death Index database was also used to determine mortality through November 2013. When the cause of death was not known, the death was categorized as cardiac. CR performed within 90 days following index REGA MPI were considered to be triggered by the MPI findings, and were censored while determining the hazard for late CR i.e., >90 days post MPI.

\section{Statistical Analysis}

Continuous data were expressed as mean \pm standard deviation. Since the MPI findings (LVEF, PDS, extent of ischemia and scar) were not normally distributed, they are shown as mean \pm standard deviation as well as median (Q1, Q3). Continuous variables are compared using analysis of variance (ANOVA). Categorical data were displayed as frequencies and percentages, and comparisons were made using Chi square tests. Survival curves were constructed for the various Groups using the Kaplan-Meier method and differences among survival curves were estimated by the log-rank test. Survival analysis treated the time of MPI as "time 0." Cox proportional hazards models were used to estimate the unadjusted and adjusted (multi-variate) hazard ratios (HRs) and 95\% confidence intervals (CIs) comparing outcome events in the different Groups. Models were constructed adjusting for demographics (age, gender, and race), co-morbidities (diabetes, hypertension, dyslipidemia, end-stage renal disease [ESRD], MI), behavioral characteristics (current smoking), prior CR, and depressed LVEF by MPI. All tests were 2-tailed, and a p value $<0.05$ was considered statistically significant. All statistical analyses were carried out with SPSS Statistics version 17 (SPSS, Inc., Chicago, Illinois). 


\section{RESULTS}

The mean age of the cohort was $61 \pm 12$ years, $58 \%$ were men, and $66 \%$ Caucasian. Almost one-half (45\%) had diabetes, $81 \%$ hypertension, 59\% dyslipidemia and 26\% ESRD. Onethird (35\%) had a history of prior CR, $18 \%$ had a history of MI, and $21 \%$ had a history of heart failure. The majority of patients reported use of cardiac medications (60\% betablockers, 55\% ACE-I or ARBs, and 54\% statin medications).

Compared to the cohort with normal REGA MPI, those with abnormal perfusion were older, more likely to be men and Caucasian, and more likely to have diabetes, dyslipidemia, and prior MI, heart failure, and CR (Table 1). Use of cardiac medications (except for calcium channel blockers) was higher in this group as well. The size of the perfusion defect, ischemia and scar and LVEF for the 4 Groups are shown in Table 1. Significantly, the proportion of patients with abnormal LVEF increased with increasing PDS reaching $61 \%$ in Group 4.

During the first 90 days after index MPI, 111 (8\%) patients had CR (35 CABG, 76 PCI). In Group 1 (normal REGA MPI), only 3 patients $(0.4 \%)$ underwent early CR. The proportion of patients who underwent early CR progressively increased with increasing PDS to reach $17 \%$ in Group 4 (PDS $>20 \%$, Figure 1). When the cohort was examined in relation to the extent of ischemia, a similar stepwise relationship was seen: 6 patients $(0.7 \%)$ in Group 5 (no ischemia) up to $29 \%$ in Group 8 (>20\% ischemia). This data confirmed that extent of PDS and ischemia on MPI is a significant driver of CR in the near term.

The patients were followed up for $46 \pm 18$ months. During follow-up, 326 (23\%) patients experienced the primary outcome; cardiac death in 231 patients (17\%), MI in 62 patients (4\%), and late CR in 90 patients (6\%). The primary outcome $(10.3 \%, 26.8 \%, 31.3 \%, 43.4 \%$, $P<.001)$, cardiac death $(7.3 \%, 17.2 \%, 22.6 \%, 31.3 \%, P<.001)$, MI $(1.3 \%, 6.4 \%, 7.7 \%$, $8.0 \%, P<.001)$, and late CR $(2.9 \%, 9.6 \%, 9.7 \%, 10.3 \%, P<.001)$ increased with increasing PDS (percentages are for Groups 1-4, respectively). Figure 2 shows the Kaplan-Meier survival curves for the primary outcome and its individual components stratified by Groups $1-4$.

Of the entire cohort, 894 (64\%) had no ischemia on REGA MPI (Group 5), 198 (14\%) had ischemia involving <10\% of LV myocardium (Group 6), 193 (14\%) 10\%-20\% (Group 7), and $115(8 \%)>20 \%$ (Group 8). The primary outcome $(16.2 \%, 29.3 \%, 39.4 \%, 40.9 \%, P<$. 001), cardiac death $(11.2 \%, 19.7 \%, 28.0 \%, 33.3 \%, P<.001)$, MI $(2.8 \%, 6.1 \%, 8.3 \%, 7.8 \%$, $P=.001)$, and late $\mathrm{CR}(4.6 \%, 10.1 \%, 10.4 \%, 7.8 \%, P=.002)$ increased with increasing extent of ischemia (percentages are for Groups 5-8, respectively). Figure 3 shows the Kaplan-Meier survival curves for the primary outcome and its individual components stratified by Groups 5-8.

To estimate the hazard ratios associated with PDS, we constructed Cox proportional hazard models for the composite primary outcome and its individual components adjusting for baseline characteristics including demographics, co-morbidities, behavioral characteristics, and prior CR (Table 2). There was an independent stepwise increase in events with increasing PDS extent that reflected a stepwise increase in cardiac death, MI, and late CR 
with increasing PDS. Depressed LVEF $(<50 \%)$ was independently associated with cardiac death but not with MI or late CR. When PDS was included in the model as a continuous variable, it was independently associated with the primary outcome (hazard ratio 1.023 [1.014-1.031]), cardiac death (1.026 [1.015-1.036]), MI (1.036 [1.017-1.055], and late CR (1.019 [1.002-1.036]). Similarly, when extent of ischemia was substituted for PDS, it was independently associated with the primary outcome (1.015 [1.006-1.025]), cardiac death (1.019 [1.008-1.030]), MI (1.027 [1.005-1.049]), and late CR (1.024 [1.004-1.044]). When LVEF was included in the model as a continuous variable, it was independently associated with the primary outcome $(0.981$ [0.972-0.990]) and cardiac death $(0.974$ [0.964-0.985) but not with MI (0.998 [0.979-1.018]), or late CR (0.992 [0.976-1.008]).

\section{DISCUSSION}

The major finding of this study is that REGA MPI provides important prognostic information that can guide clinical management. The perfusion findings on REGA MPI informed decision-making regarding $\mathrm{CR}$ in the near term (first 90 days) and were independently associated with cardiac outcomes including cardiac death, MI, and CR in the long term (several years). Further, LVEF by gated SPECT provided incremental prognostic information to the perfusion data and clinical characteristics with regard to cardiac mortality. Lately, there has been growing concern regarding the incremental value that advanced cardiovascular imaging provides to patient care especially with its associated high cost to society. ${ }^{11}$ It is paramount that the incremental value provided by imaging is well established to support its use. This is particularly important in the case of MPI since several million scans are performed on an annual basis in the US. The prognostic utility of MPI using older generation vasodilators, such as adenosine and dipyridamole, is supported by a compelling body of literature. ${ }^{6,12-17}$ However, up to our knowledge, the prognostic data provided by REGA MPI have not been examined in depth.

In contrast to adenosine, REGA is less rapidly metabolized allowing its administration as a fixed-dose bolus. ${ }^{18}$ Following bolus administration, the peak plasma concentration of REGA is achieved within 1-3 minutes. In dogs, REGA induces a dose-dependent increase in coronary blood flow and is as effective but more potent than adenosine and its effect is longer lasting. ${ }^{19}$ In humans, REGA increased peak blood flow velocity by up to 3.4-fold in a dose-dependent manner. ${ }^{18}$ The peak blood velocities are achieved within $\sim 2$ minutes and the mean duration of the increase in flow velocity of twofold or greater is 8.5 minutes. ${ }^{18}$ The increase in coronary blood flowing by REGA was on average $84 \%$ of the maximal reactive hyperemia following coronary occlusion. Since perfusion defects by MPI are thought to reflect disparities in coronary blood flow between regions supplied by diseased compared to normal coronary arteries, these data suggest that coronary hyperemia induced by REGA is suitable for assessing perfusion abnormalities by MPI. Indeed, in the multi-center ADVANCE MPI (Adenosine vs Regadenoson Comparative Evaluation for Myocardial Perfusion Imaging) trials, REGA was shown to be non-inferior to adenosine for diagnosis of reversible perfusion defects. ${ }^{2,3,20}$ However, Advance MPI did not provide any data on prognostic value of REGA. 
An important function of MPI is to act as a gate keeper for coronary angiography. There has been increasing attention to the inappropriate use of coronary angiography and PCI. For example, in a recent study of 544 hospitals involving more than a million patients undergoing elective coronary angiography, $25 \%$ were asymptomatic. ${ }^{21}$ Further, performance of coronary angiography in asymptomatic patients was associated with higher rates of inappropriate PCI. In this study, only $36 \%$ of patients undergoing elective coronary angiography were intermediate-high risk by non-invasive testing (33\% did not undergo testing and 30\% were low risk). In another recent study from New York State, 25\% of patients undergoing diagnostic catheterization were rated inappropriate and of those, approximately two-third had no previous stress test. ${ }^{22}$ This data complement earlier studies that reported that the majority of patients undergoing elective coronary angiography in the US do not have obstructive CAD. ${ }^{23}$ The current appropriate use criteria favors the use of non-invasive testing as a gate keeper for invasive angiography especially in asymptomatic patients and in symptomatic patients with low or intermediate pretest probability. ${ }^{24} \mathrm{An}$ important question faced by clinicians who request MPI for their patients is whether the results warrant further investigation usually with invasive angiography and, if appropriate, CR. In our retrospective study, an increasing PDS and area of ischemia were associated with higher rates of proceeding with CR in the short term. Further, the association of the degree of perfusion abnormality with events in the long term also lends support to the use of REGA MPI to help with decision-making. Of particular interest is the very low rate of early $\mathrm{CR}$ in patients with normal REGA MPI $(0.7 \%)$ and the low rate of events during follow-up in these patients.

The data presented here complement our previous report in patients with normal perfusion. ${ }^{7}$ Our previous study demonstrated that a REGA MPI is as effective as adenosine MPI in identifying patients at low risk of cardiovascular events. However, since the study was conducted exclusively in patients with normal perfusion, we were unable to show the risk associated with an abnormal study. In the current study, we show that the cardiovascular risk increases stepwise with increasing size of the perfusion defect and with increasing extent of ischemia. This risk was independent of baseline characteristics, co-morbidities, and cardiac history. For example, a large perfusion defect $(>20 \% \mathrm{LV}$ ) was associated with a $>$ fourfold increased risk of cardiac death, >eightfold increased risk of MI, and fourfold increased risk of late CR compared to patients with no perfusion defect. When analyzed as a continuous factor, a 10\% increase in PDS was independently associated with a $26 \%$ increase risk of cardiac death, $36 \%$ increased risk of MI, and $19 \%$ increased risk of late CR. In addition, a depressed LVEF ( $<50 \%$ ) was independently associated with a $42 \%$ increased risk of cardiac death. These data demonstrate that REGA MPI provides powerful prognostic data that are incremental to that provided by clinical characteristics.

Our study has many limitations that should be considered. First, the study is retrospective and the scan findings were available to the treating physicians and likely have influenced decision-making. It is difficult to make a determination whether a diagnostic test finding altered therapy in a retrospective study. We used the conventional cut-off of 90 days for CR driven by MPI which may have led to increased late $\mathrm{CR}$ procedures which in fact were due to MPI findings. However, inspection of the Kaplan-Meier graph for late CR (Figure 2D) demonstrates that the event rates for normal and abnormal MPIs continue to diverge even 
years after index MPI. Further, findings from MPI may have led to intensification of medical therapy (which is not captured in our study) which would have decreased the magnitude of difference between the groups. Second, our study is from a single tertiary care academic center, and therefore our findings may not be generalizable. Patients undergoing vasodilator MPI are usually at higher risk for adverse events than those undergoing exercise MPI, likely due to the presence of multiple co-morbidities. ${ }^{25}$ Also, our results are from a transplant center that uses MPI for the evaluation of kidney transplant candidates; a quarter of our population had ESRD which is known to be at elevated risk. ${ }^{26,27}$ In our analysis, ESRD was associated with increased cardiac mortality independent of other characteristics, such as diabetes, and of REGA MPI findings (Table 2). These data are supportive of a recent report by Bhatti et al ${ }^{28}$ that showed that renal function is a powerful risk predictor in patients undergoing REGA MPI. Lastly, due to the difficulty of categorizing deaths as cardiac or non-cardiac in retrospective non-adjudicated studies, we performed a secondary analysis using all-cause death instead of cardiac death and the findings remained unchanged (with higher event rates). Nevertheless, this is the first study that examines the prognostic value of abnormal REGA MPI in a non-selected population.

\section{NEW KNOWLEDGE GAINED}

The myocardial perfusion pattern by REGA MPI informs decision-making with regard to $\mathrm{CR}$ and is independently associated with cardiac death, MI, and CR over several years. In addition, LVEF by gated SPECT adds prognostic information to the perfusion pattern with regard to cardiac death. REGA MPI provides powerful prognostic information that has important implications in patient management and can guide clinical practice.

\section{Acknowledgments}

\section{Disclosures}

Drs Hage and Iskandrian have received research grants from Astellas Pharma USA.

\section{References}

1. Ghimire G, Hage FG, Heo J, Iskandrian AE. Regadenoson: A focused update. J Nucl Cardiol. 2013; 20:284-288. [PubMed: 23229649]

2. Iskandrian AE, Bateman TM, Belardinelli L, Blackburn B, Cerqueira MD, Hendel RC, et al. Adenosine versus regadenoson comparative evaluation in myocardial perfusion imaging: Results of the ADVANCE phase 3 multicenter international trial. J Nucl Cardiol. 2007; 14:645-658. [PubMed: 17826318]

3. Cerqueira MD, Nguyen P, Staehr P, Underwood SR, Iskandrian AE. Effects of age, gender, obesity, and diabetes on the efficacy and safety of the selective A2A agonist regadenoson versus adenosine in myocardial perfusion imaging integrated ADVANCE-MPI trial results. JACC Cardiovasc Imaging. 2008; 1:307-316. [PubMed: 19356442]

4. 2013 Nuclear Cardiology Trend Survey. J Nucl Cardiol. 2014; 21:S5-S88.

5. Zoghbi GJ, Iskandrian AE. Selective adenosine agonists and myocardial perfusion imaging. J Nucl Cardiol. 2012; 19:126-141. [PubMed: 22130964]

6. Shaw LJ, Hage FG, Berman DS, Hachamovitch R, Iskandrian A. Prognosis in the era of comparative effectiveness research: Where is nuclear cardiology now and where should it be? $\mathrm{J}$ Nucl Cardiol. 2012; 19:1026-1043. [PubMed: 22760523] 
7. Iqbal FM, Hage FG, Ahmed A, Dean PJ, Raslan S, Heo J, et al. Comparison of the prognostic value of normal regadenoson with normal adenosine myocardial perfusion imaging with propensity score matching. JACC Cardiovasc Imaging. 2012; 5:1014-1021. [PubMed: 23058068]

8. Henzlova MJ, Cerqueira MD, Hansen CL, Taillefer R, Yao SS. Stress protocols and tracers. ASNC imaging guidelines for nuclear cardiology procedures. J Nucl Cardiol. 2009

9. Ficaro EP, Lee BC, Kritzman JN, Corbett JR. Corridor4DM: The Michigan method for quantitative nuclear cardiology. J Nucl Cardiol. 2007; 14:455-465. [PubMed: 17679053]

10. Germano G, Kiat H, Kavanagh PB, Moriel M, Mazzanti M, Su HT, et al. Automatic quantification of ejection fraction from gated myocardial perfusion SPECT. J Nucl Med. 1995; 36:2138-2147. [PubMed: 7472611]

11. Mark DB, Anderson JL, Brinker JA, Brophy JA, Casey DE Jr, Cross RR, et al. ACC/AHA/ASE/ ASNC/HRS/IAC/Mended Hearts/NASCI/RSNA/SAIP/SCAI/SCCT/SCMR/SNMMI 2014 health policy statement on use of noninvasive cardiovascular imaging: A report of the American College of Cardiology Clinical Quality Committee. J Am Coll Cardiol. 2014; 63:698-721. [PubMed: 24556329]

12. Hachamovitch R, Berman DS, Kiat H, Cohen I, Lewin H, Amanullah A, et al. Incremental prognostic value of adenosine stress myocardial perfusion single-photon emission computed tomography and impact on subsequent management in patients with or suspected of having myocardial ischemia. Am J Cardiol. 1997; 80:426-433. [PubMed: 9285653]

13. Amanullah AM, Berman DS, Erel J, Kiat H, Cohen I, Germano G, et al. Incremental prognostic value of adenosine myocardial perfusion single-photon emission computed tomography in women with suspected coronary artery disease. Am J Cardiol. 1998; 82:725-730. [PubMed: 9761081]

14. Hendel RC, Layden JJ, Leppo JA. Prognostic value of dipyridamole thallium scintigraphy for evaluation of ischemic heart disease. J Am Coll Cardiol. 1990; 15:109-116. [PubMed: 2295718]

15. Kamal AM, Fattah AA, Pancholy S, Aksut S, Cave V, Heo J, et al. Prognostic value of adenosine single-photon emission computed tomographic thallium imaging in medically treated patients with angiographic evidence of coronary artery disease. J Nucl Cardiol. 1994; 1:254-261. [PubMed: 9420708]

16. Navare SM, Mather JF, Shaw LJ, Fowler MS, Heller GV. Comparison of risk stratification with pharmacologic and exercise stress myocardial perfusion imaging: A meta-analysis. J Nucl Cardiol. 2004; 11:551-561. [PubMed: 15472640]

17. Hachamovitch R, Hayes SW, Friedman JD, Cohen I, Berman DS. A prognostic score for prediction of cardiac mortality risk after adenosine stress myocardial perfusion scintigraphy. $\mathrm{J}$ Am Coll Cardiol. 2005; 45:722-729. [PubMed: 15734617]

18. Lieu HD, Shryock JC, von Mering GO, Gordi T, Blackburn B, Olmsted AW, et al. Regadenoson, a selective A2A adenosine receptor agonist, causes dose-dependent increases in coronary blood flow velocity in humans. J Nucl Cardiol. 2007; 14:514-520. [PubMed: 17679059]

19. Trochu JN, Zhao G, Post H, Xu X, Belardinelli L, Belloni FL, et al. Selective A2A adenosine receptor agonist as a coronary vasodilator in conscious dogs: Potential for use in myocardial perfusion imaging. J Cardiovasc Pharmacol. 2003; 41:132-139. [PubMed: 12500031]

20. Mahmarian JJ, Peterson LE, Xu J, Cerqueira MD, Iskandrian AE, Bateman TM, et al. Regadenoson provides perfusion results comparable to adenosine in heterogeneous patient populations: A quantitative analysis from the ADVANCE MPI trials. J Nucl Cardiol. 2014

21. Bradley SM, Spertus JA, Kennedy KF, Nallamothu BK, Chan PS, Patel MR, et al. Patient selection for diagnostic coronary angiography and hospital-level percutaneous coronary intervention appropriateness: Insights from the national cardiovascular data registry. JAMA Intern Med. 2014; 174:1630-1639. [PubMed: 25156821]

22. Hannan EL, Samadashvili Z, Cozzens K, Walford G, Holmes DR Jr, Jacobs AK, et al. Appropriateness of diagnostic catheterization for suspected coronary artery disease in New York State. Circ Cardiovasc Interv. 2014; 7:19-27. [PubMed: 24474625]

23. Patel MR, Peterson ED, Dai D, Brennan JM, Redberg RF, Anderson HV, et al. Low diagnostic yield of elective coronary angiography. N Engl J Med. 2010; 362:886-895. [PubMed: 20220183]

24. Patel MR, Bailey SR, Bonow RO, Chambers CE, Chan PS, Dehmer GJ, et al. ACCF/SCAI/ AATS/AHA/ASE/ASNC/HFSA/HRS/SCCM/SCCT/SCMR/STS 2012 appropriate use criteria for 
diagnostic catheterization: A report of the American College of Cardiology Foundation Appropriate Use Criteria Task Force, Society for Cardiovascular Angiography and Interventions, American Association for Thoracic Surgery, American Heart Association, American Society of Echocardiography, American Society of Nuclear Cardiology, Heart Failure Society of America, Heart Rhythm Society, Society of Critical Care Medicine, Society of Cardiovascular Computed Tomography, Society for Cardiovascular Magnetic Resonance, and Society of Thoracic Surgeons. J Am Coll Cardiol. 2012; 59:1995-2027. [PubMed: 22578925]

25. Rozanski A, Gransar H, Hayes SW, Friedman JD, Hachamovitch R, Berman DS. Comparison of long-term mortality risk following normal exercise vs adenosine myocardial perfusion SPECT. $\mathrm{J}$ Nucl Cardiol. 2010; 17:999-1008. [PubMed: 21076898]

26. Hage FG, Venkataraman R, Zoghbi GJ, Perry GJ, DeMattos AM, Iskandrian AE. The scope of coronary heart disease in patients with chronic kidney disease. J Am Coll Cardiol. 2009; 53:21292140. [PubMed: 19497438]

27. Rizk DV, Riad S, Hage FG. Screening for coronary artery disease in kidney transplant candidates. J Nucl Cardiol. 2014

28. Bhatti S, Hakeem A, Dhanalakota S, Palani G, Husain Z, Jacobsen G, et al. Prognostic value of regadenoson myocardial single-photon emission computed tomography in patients with different degrees of renal dysfunction. Eur Heart J Cardiovasc Imaging. 2014; 15:933-940. [PubMed: 24699324] 


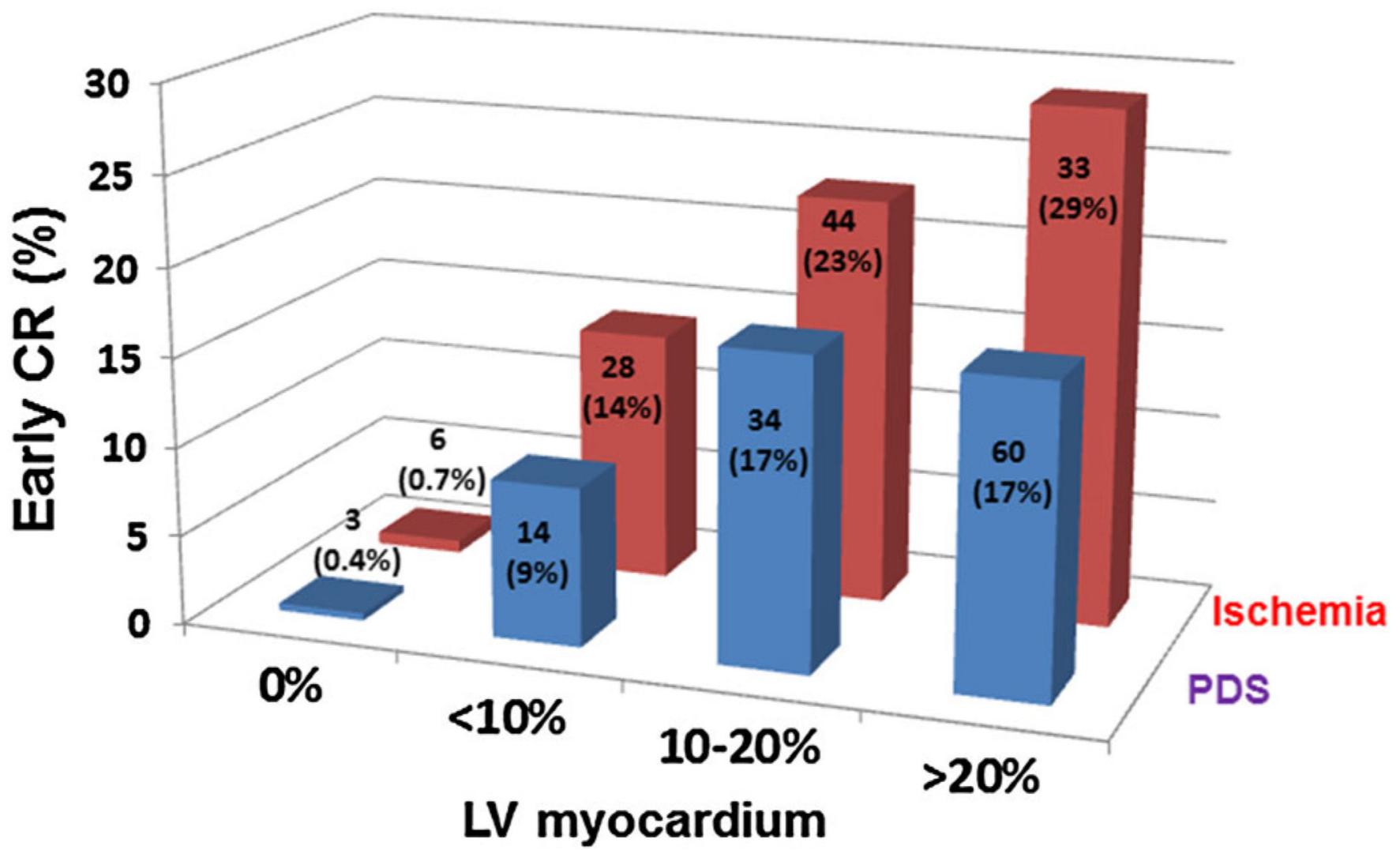

Figure 1.

Incidence of coronary revascularization (CR) within 90 days of index MPI (early CR) according to PDS and extent of ischemia expressed as \% of LV. The number in each bar represents the number of patients in each category who underwent early $\mathrm{CR}$ followed by the percentage that represents of each category in parentheses. Chi square $P<.001$ for both. CR, coronary revascularization; LV, left ventricle; PDS, perfusion defect size. 
A Cardiac Death, Myocardial Infarction or late Coronary Revascularization

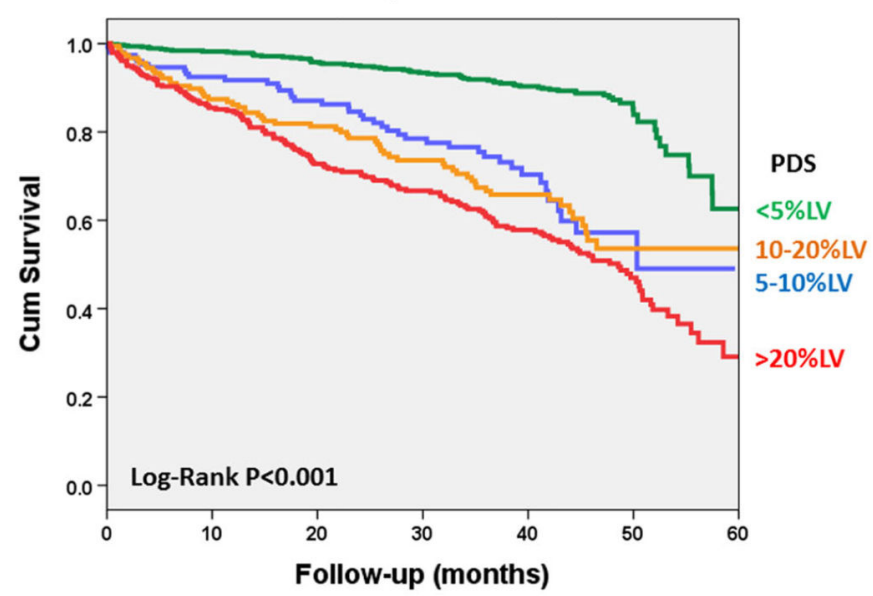

C

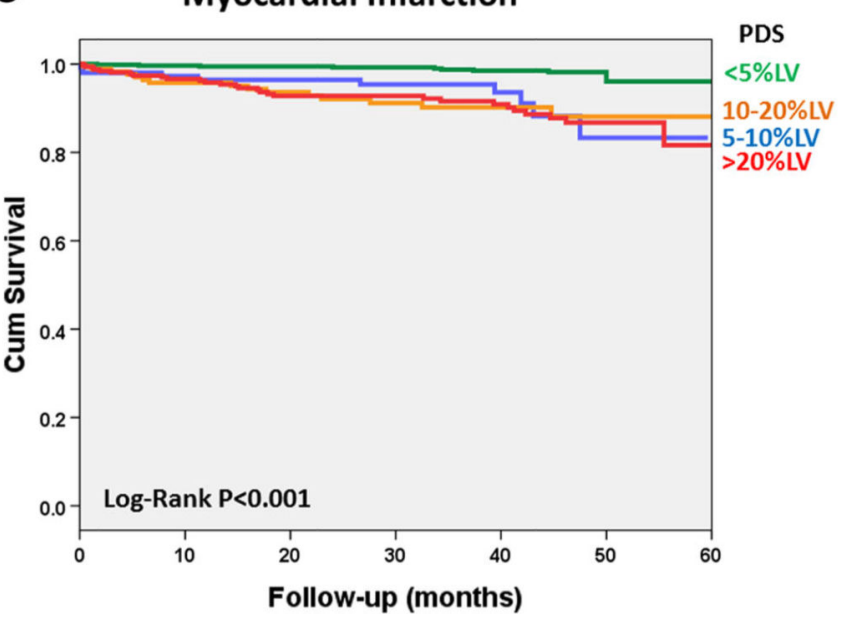

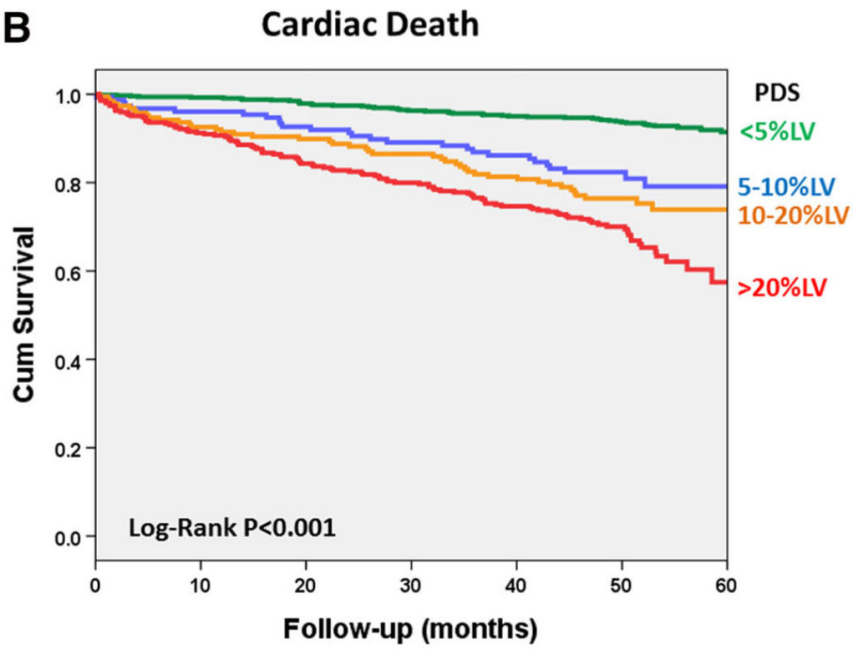

D Late Coronary Revascularization

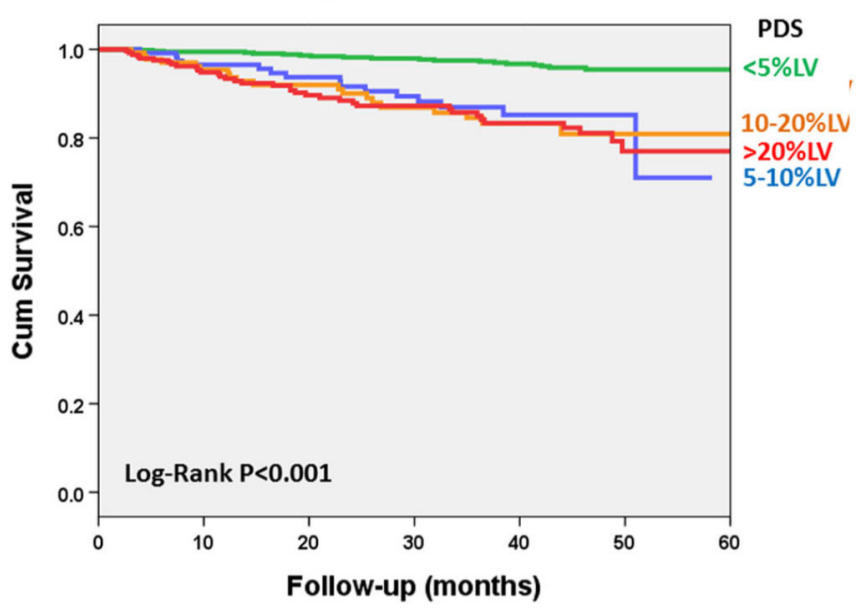

Figure 2.

Kaplan-Meier survival curves for the composite outcome of cardiac death, MI, and late CR (A), cardiac death (B), MI (C), and late CR (D) stratified by PDS categories. 


\section{A Cardiac Death, Myocardial Infarction}

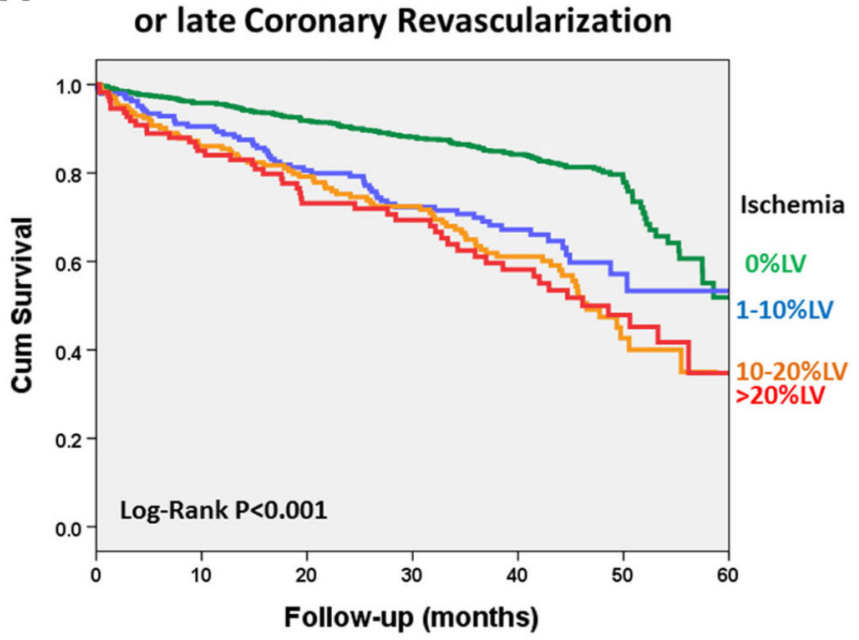

C

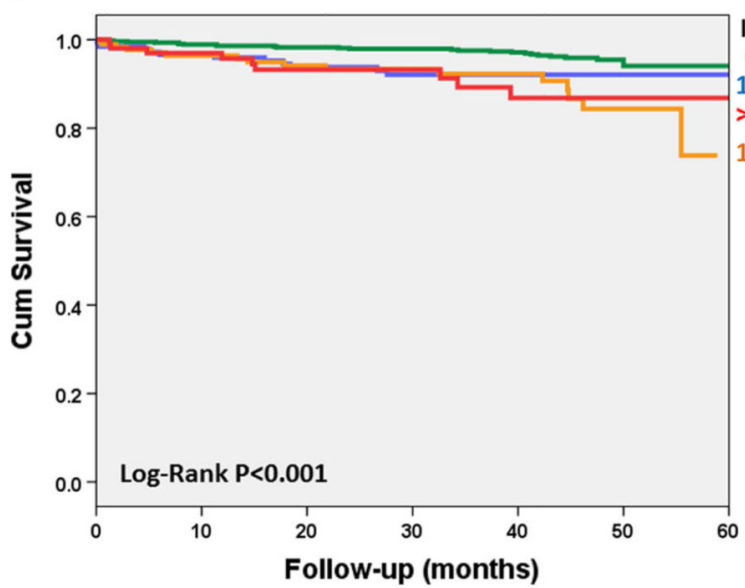

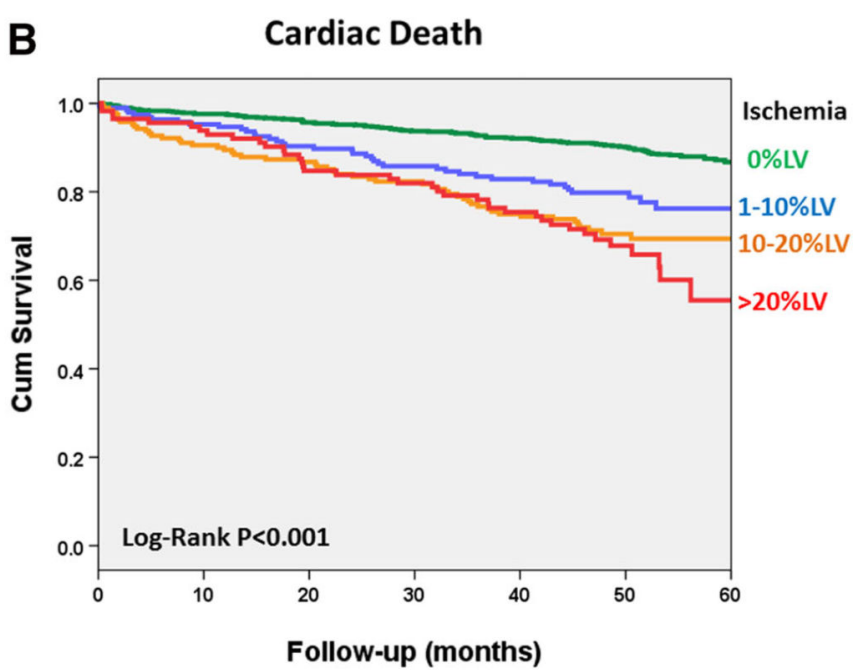

D Late Coronary Revascularization

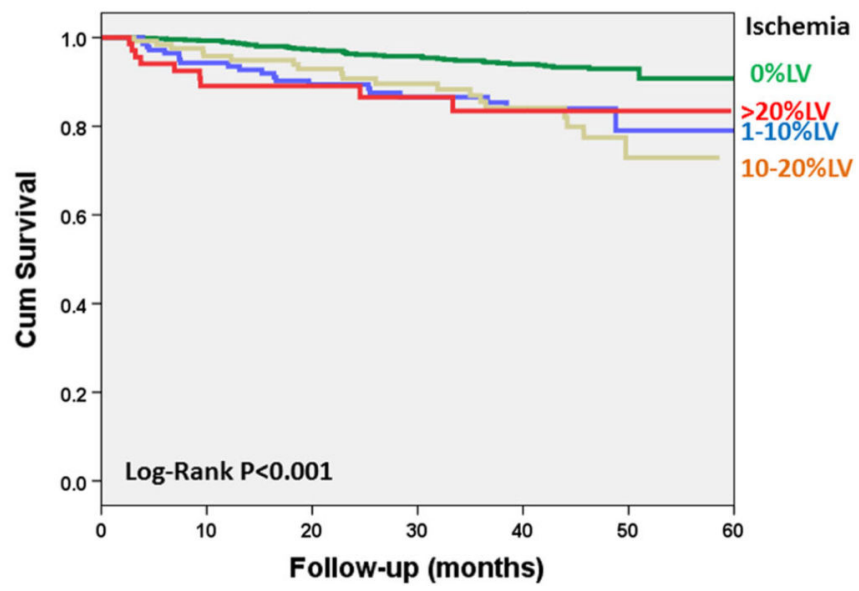

Figure 3.

Kaplan-Meier survival curves for the composite outcome of cardiac death, MI, and late CR (A), cardiac death (B), MI (C), and late CR (D) stratified by ischemia categories. 


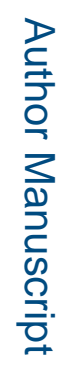

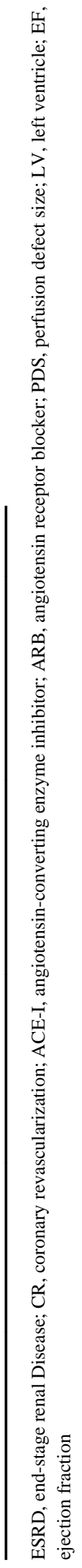

를

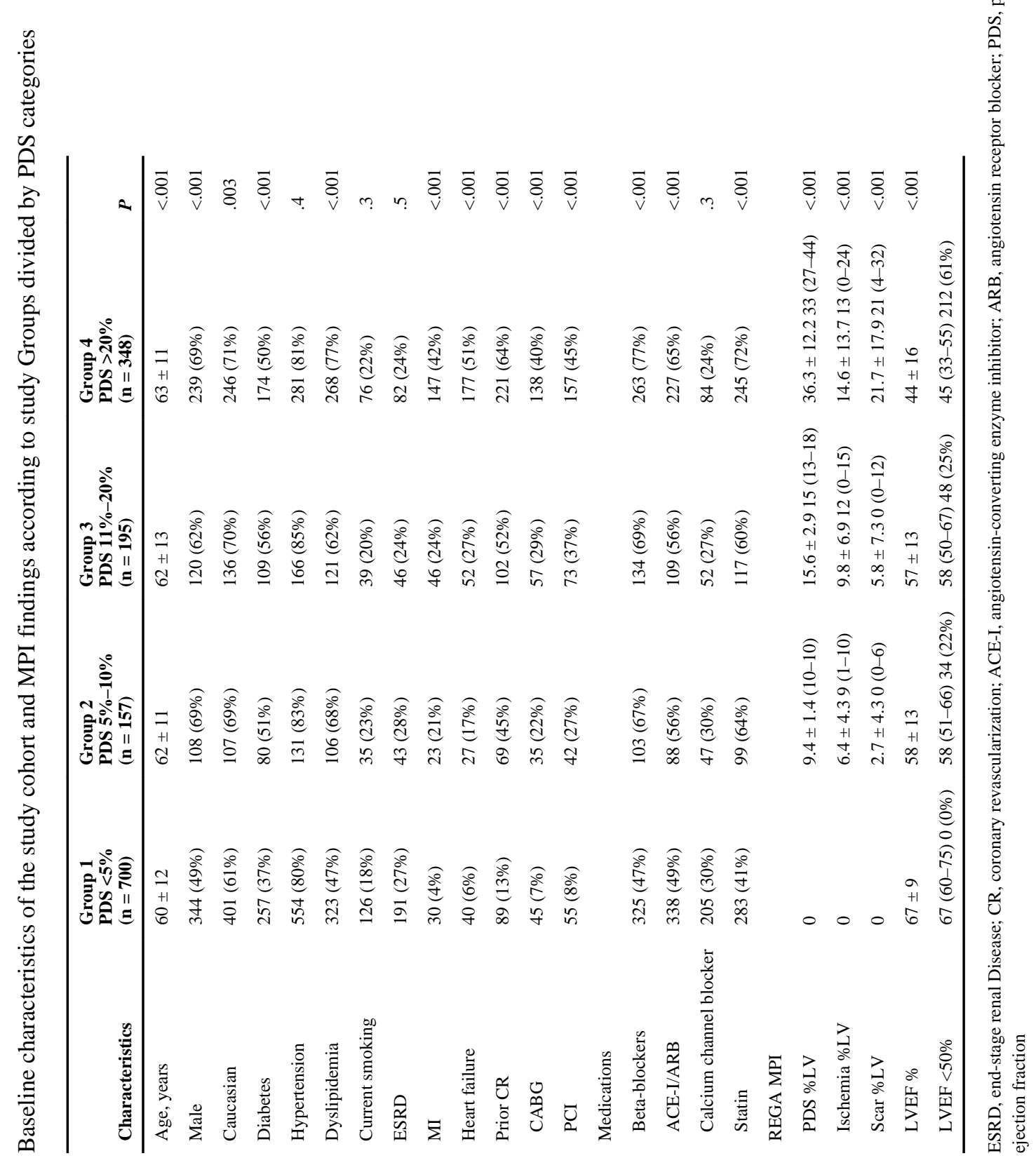
J Nucl Cardiol. Author manuscript; available in PMC 2016 December 01. 


\section{Table 2}

Cox proportional hazard ratio for the primary outcome and its components according to PDS categories

\begin{tabular}{|c|c|c|c|c|}
\hline Variable & Primary outcome & Cardiac death & MI & Late CR \\
\hline Age (years) & $1.008(0.997-1.019)$ & $1.022(1.009-1.036)$ & $1.006(0.981-1.030)$ & $0.977(0.958-0.998)$ \\
\hline Male gender & $1.142(0.895-1.457)$ & $1.224(0.910-1.646)$ & $0.964(0.557-1.670)$ & $1.124(0.705-1.791)$ \\
\hline Caucasian race & $0.768(0.599-0.984)$ & $0.803(0.596-1.082)$ & $1.016(0.569-1.815)$ & $1.207(0.725-2.008)$ \\
\hline Diabetes & $1.396(1.102-1.769)$ & 1.485 (1.116-1.976) & $1.810(1.058-3.097)$ & $1.322(0.853-2.049)$ \\
\hline Hypertension & $0.762(0.561-1.035)$ & $0.818(0.564-1.187)$ & $0.749(0.468-1.918)$ & $0.800(0.469-1.365)$ \\
\hline Dyslipidemia & $0.676(0.524-0.873)$ & $0.629(0.468-0.846)$ & $0.594(0.337-1.048)$ & $0.672(0.408-1.109)$ \\
\hline ESRD & $2.050(1.565-2.686)$ & $2.939(2.141-4.034)$ & $1.214(0.623-2.363)$ & $1.236(0.699-2.187)$ \\
\hline Prior MI & $0.910(0.691-1.197)$ & $0.875(0.627-1.219)$ & $0.846(0.458-1.564)$ & $0.866(0.518-1.448)$ \\
\hline Current smoker & $1.303(0.980-1.731)$ & $1.171(0.827-1.658)$ & $1.603(0.860-2.990)$ & $1.634(0.990-2.698)$ \\
\hline Prior CR & $1.508(1.158-1.963)$ & $1.403(1.025-1.920)$ & $1.206(0.678-2.146)$ & $2.474(1.431-4.276)$ \\
\hline \multicolumn{5}{|l|}{ PDS } \\
\hline Normal perfusion & Ref & Ref & Ref & Ref \\
\hline$<10 \% \mathrm{LV}$ & $2.682(1.772-4.061)$ & $2.489(1.488-4.164)$ & $5.765(2.249-14.778)$ & $3.518(1.704-7.263)$ \\
\hline $10-20 \% \mathrm{LV}$ & $3.322(2.284-4.832)$ & $3.408(2.180-5.327)$ & $6.720(2.788-16.198)$ & 3.925 (1.949-7.907) \\
\hline$>20 \% \mathrm{LV}$ & $4.050(2.775-5.910)$ & $4.588(2.904-7.248)$ & $8.250(3.357-20.278)$ & 4.078 (1.939-8.575) \\
\hline LVEF $<50 \%$ & $1.185(0.897-1.565)$ & $1.419(1.021-1.972)$ & $0.836(0.452-1.548)$ & $1.062(0.619-1.822)$ \\
\hline
\end{tabular}

The adjusted hazard ratio for each variable and its $95 \%$ confidence interval is shown. Variables that are statistically significant are shown in bold. 